Voix et Images

voixetimages

\title{
Le journal intime comme fiction
}

\section{André Carpentier}

Volume 15, numéro 1 (43), automne 1989

Jacques Poulin

URI : https://id.erudit.org/iderudit/200823ar

DOI : https://doi.org/10.7202/200823ar

Aller au sommaire du numéro

Éditeur(s)

Université du Québec à Montréal

ISSN

0318-9201 (imprimé)

1705-933X (numérique)

Découvrir la revue

Citer cet article

Carpentier, A. (1989). Le journal intime comme fiction. Voix et Images, 15(1),

121-124. https://doi.org/10.7202/200823ar

Ce document est protégé par la loi sur le droit d'auteur. L'utilisation des services d'Érudit (y compris la reproduction) est assujettie à sa politique d'utilisation que vous pouvez consulter en ligne.

https://apropos.erudit.org/fr/usagers/politique-dutilisation/
Cet article est diffusé et préservé par Érudit.

Érudit est un consortium interuniversitaire sans but lucratif composé de l’Université de Montréal, l'Université Laval et l'Université du Québec à Montréal. Il a pour mission la promotion et la valorisation de la recherche. https://www.erudit.org/fr/ 


\section{Le journal intime comme fiction}

\section{par André Carpentier, Université du Québec à Montréal}

[Dès avant que d'aborder la théorie appliquée au journal intime, s'épigraphier à ce mot de Saint-Denys Garneau, daté du 15 avril 1935: Que le grand art consiste à dépasser la réalité et non à la fuir. Il faut qu'on puisse dire: «comment c'est cela; et quelque chose de plus.» C'est dans ce «plus» que réside l'art. ${ }^{1}$ ]

C'est un fait d'observation que la critique constitue une observation après le fait. Une réflexion menée à distance variable de l'observé, dira-t-on. Or, l'intérêt récent des analystes de la scène littéraire pour la littérature personnelle (autobiographie, mémoires, correspondance, journal intime...), et en particulier pour la composante diaristique, paraît se conjoindre avec un peu de retard au développement du genre intime chez les écrivains et les écrivaines depuis quelques décennies. Le journal intime au Québec. Structure, évolution, réception ${ }^{2}$, de Pierre Hébert, avec la collaboration de Marilyn Baszczynski, veut aider à combler ce décalage en introduisant à une mise en situation du genre intime au Québec et en proposant une contribution à une typologie du journal; cela n'est pas rien, surtout si l'on considère que les travaux sur le journal intime québécois sont très peu nombreux et que les études narratologiques sur le journal en général - c'est ce qui nous est proposé dans les meilleures pages de cet ouvrage - sont plus rares encore. Au plan historique, l'objectif est de cerner le processus d'évolution du genre au Québec entre les années 1881 et 1980 (le corpus comprend soixante-cinq journaux, dont trente-neuf furent publiés après 1960), par l'observation de la réception critique et de l'évolution de la notion de subjectivité - au Québec toujours; au plan générique, Pierre Hébert ajoute quelques jalons à une narratologie appliquée depuis peu au journal intime, développant parallèlement des traits généraux et des considérations sur des corpus particuliers.

Dans les premiers pas de la démarche, le journal est abordé par l'examen de positions développées dans des études qui font autorité. L'objet de ce regard critique, quelque peu rapide il faut dire, est de proposer les prémices d'une typologie basée sur deux régulations fondamentales, la mimésis et la diégésis, la mimésis se définissant par un maximum d'information et un minimum $d$ 'informateur, et la diégésis par le rapport inverse ${ }^{3}$. En simple, Hébert pose la question des rapports que le narrateur du journal entretient avec ce qu'il raconte, il scrute son attitude, et sa compétence - en termes de vouloir, de pouvoir et de savoir; car c'est sur cette modalité, le degré d'intervention du narrateur dans la sélection et la présentation des faits (p. 26), que se fonde son approche héritée de la sémiotique littéraire et surtout de la narratologie. Cela revient à s'interroger sur le statut du récit (ce mot pris au sens courant d'un discours qui assume la relation d'un événement) dans le journal intime. Pour tout dire, c'est surtout sur cette question, qui trouvera ses développements les plus féconds dans la seconde partie du livre, que les travaux de Pierre Hébert paraissent apporter une contribution originale aux études sur le journal. 
Le détour obligé par les questions de réception et d'histoire de la subjectivité pousse Hébert à l'ébauche d'une périodisation qui lui permet d'esquisser la progression de la subjectivité et les transformations de l'idée qu'on se fait du journal intime au Québec durant la période couverte par l'étude. C'est vers 1840 que le journal se constitue comme genre, mais à cette date, et jusque vers 1930, il n'a qu'une fonction utilitaire, et le moi, pour reprendre un sous-titre de l'ouvrage, demeure derrière les coulisses (sic, p. 54). La réception est conséquente à ce moi occulté: on constate une absence de réflexion sur le journal. Les dates clés de l'histoire du journal intime au Québec, qui ouvrent et ferment en quelque sorte la seconde période (le moi-recouvré), correspondent à peu près au début de la rédaction et à la parution du Journal de Saint-Denys Garneau 4: avant Saint-Denys Garneau, on ne voit pas exactement à quoi sert le journal (p. 44); avec lui, le diariste affirme que son centre, sa seule certitude demeure le moi (p. 62). Il résultera de cette parution une réflexion-débat entre ceux qui entretiennent une trouble méfiance à l'égard de l'introspection et ceux qui se refusent à renoncer au drame de l'intériorité. La dernière période est celle du moi-affirmé, de l'expérience du conflit entre les valeurs personnelles et les ancrages extérieurs (p. 63); là, se réalise la coïncidence du sujet et de l'objet.

On notera, avec Pierre Hébert, une exception marquante, dans cette périodisation, celle d'Henriette Dessaulles, qui tient, entre 1874 et 1881 , un journal où se développe un moi glorifié; ce Journal ne sera publié qu'en 1971: la subjectivité qui s'y manifeste peut alors bénéficier du nihil obstat institutionnel (p. 71).

La première partie du livre se termine sur un ton plutôt essayistique; Hébert y soutient l'hypothèse, productive mais qui nécessiterait d'être étayée, d'un rapport étroit entre les trois stades du moi et l'évolution des idéologies au Québec depuis 1840; il se propose d' homologuer [ses] conclusions sur l'évolution de la subjectivité dans le journal aux déterminismes (relatifs) imposés par la situation coloniale (p. 77). À une extrémité du tableau: le moi masqué, auquel correspond une subordination du moi au «nous» de la solidarité; bien sûr, les conditions culturelles nécessaires à l'éclosion du journal ne sont pas réunies. À l'autre extrémité, au stade de l'émancipation du moi: un besoin de retour sur soi et de compréhension des démarches individuelles et collectives 5 . Entre les deux, le recouvrement du moi: Comme la vérité ne se prononce plus dans le nous, un nouvel étalon des valeurs s'articule dans l'individu qui doit rapatrier la norme de son espace intérieur. (p. 78)

La deuxième partie de l'ouvrage, intitulée «Pour une narratologie du journal intime», propose trois monographies qui veulent sonder des aspects textuels caractéristiques du journal intime. Dans «Le statut du récit dans le Journal d'Henriette Dessaulles», Hébert ajoute à l'approfondissement de deux concepts fondamentaux. D'abord celui de récit. Devant l'apparente absence de logique du récit dans le journal, qui serait consécutive à une absence de structure (Béatrice Didier6), Hébert élargit la notion de récit à l' histoire d'une vie se nouant et se dénouant au jour le jour (p. 88). Il revisite ainsi le principe d'antériorité de l'histoire sur le discours dans le journal: 


\section{[...] s'il y a récit dans le journal, ce n'est pas au premier niveau, celui de l'événement du jour, mais plutôt au second plan où le diariste s'approprie l'événement du premier niveau pour l'intégrer dans un discours $[\ldots]$ (p. 95).}

En ce sens, il y a récit lorsque l'événement est intégré à l'expérience intérieure. Cette distinction amène Hébert à fonder son modèle sur le concept de fonction rhématique, où le thème, affirmation de fait, est modalisé par l'attitude du locuteur - le rhème. Puis il examine de près, dans Fadette ${ }^{7}$, de Dessaulles, les différents types d'interventions visant à intégrer les thématiques dans la subjectivité de la journalière.

Plus loin, c'est la délicate question du narrataire dans le journal intime qui est soulevée, cette fois chez Lionel Groulx. Pierre Hébert augmente cette fois la vision de Jean Rousset ${ }^{8}$, qui reconnaissait deux classes de narrataires dans le journal intime : le narrataire interne: le rédacteur s'adresse à lui-même; et le narrataire externe: il s'adresse à un allocutaire non récepteur. Puis Hébert de relever plusieurs narrataires, un absent, des opposants, des narrataires rhétoriques... L'autre intérêt majeur de ce chapitre tient à l'hypothèse qui y est développée concernant l'abandon du Journal par Lionel Groulx. Au fil des ans, et surtout dans les trois premières années du siècle, s'enclenche une chute des relations intra-personnelles (avec le journal-narrataire), donc abandon progressif de la quête de soi au profit de rapports avec Dieu (avec le Dieu-narrataire), donc au bénéfice de la quête divine. Témoin, ce fragment de 1903:

$J$ 'ai presque délaissé mon journal. Le temps est venu d'occupations sérieuses, plus sérieuses. [...] Autrefois, j’avais ce besoin, besoin impérieux de vider ici mon âme. Aujourd' hui, je la vide dans mes lettres et dans l'âme des jeunes. C'est plus utile et plus prêtre. ${ }^{9}$

Le dernier chapitre du livre est de Marilyn Baszczynski et porte sur les structures actantielles dans le Journal de Saint-Denys Garneau. On y trouve une application éclairante - et même extensive - de l'approche développée dans les chapitres précédents. L'intérêt, ici, se porte sur la force des tensions si chargées d'émotion qui traversent le Journal, et sur la double quête, de soi - d'équilibre - et de Dieu, qui s'y développe.

À ces chapitres, s'aboute une bibliographie de près de cinq cents documents, dont une soixantaine relevant de la critique allemande(!), précédée d'une conclusion en forme de plaidoyer pour la fiction, où il est réitéré non pas que les événements eux-mêmes sont fictifs, mais bien que leur prise en charge par un texte relève véritablement d' une opération de mise en discours ou mieux, de mise en intrigue (p. 162). Shoshana Felman comprenait la littérature comme refoulé de la philosophie, Pierre Hébert n'est pas loin de considérer le journal intime comme refoulé de la fiction - ou n'est-ce pas le contraire?

On pourra donc mener parallèlement deux lectures de ce livre: l'une qui concerne la théorie - elle sera satisfaite par les questions qu'elle soulève -, l'autre qui s'applique au corpus - celle-là sera moins riche d'enseignement, car l'ouvrage, malgré son titre, n'a pas vraiment valeur de synthèse. Au-delà de ces 
deux parcours, on aura plaisir à fouiller les échappées de cette étude, ces zones secondaires où Hébert et Baszczynski laissent percer leur conception profonde du journal intime. Attrapons au rebond quelques-uns de ces principes, qui contrarient le goût de ceux qui voient le journal intime comme une nécrologie anticipée: la publication anthume du journal ne constitue pas un sacrilège, mais une nouvelle modalité du même champ générique (p. 13); l'historicité du journal intime ne s'établit pas sur sa cohérence rétrospective, mais sur l'avènement d'un devenir; toute vie s'énonce en différé peu importe que ce différé se calcule en micro secondes ou en années-lumières 10; le journal intime est moins un document psychologique qu'une œuvre d'écriture; le diariste est engagé dans l'écriture d'un livre, le moi textuel ${ }^{11}$ a préséance sur le moi révolu; il y a un mouvement de l'auvre (p. 43) où se lit ce que le diariste porte en lui; le lieu de l'inattendu, dans le journal, se situe moins au niveau thématique que rhématique, moins au niveau de ce dont ça parle qu'à celui de ce qui en est dit - le grand art consiste à dépasser la réalité...

1 Hector de Saint-Denys Gameau, Journal, préface de Gilles Marcotte, «Avertissement» de Robert Élie et Jean Le Moyne, Montréal, Beauchemin, 1963 [1954], p. 58.

2 Pierre Hébert, avec la collaboration de Marilyn Baszczynski, le Journal intime au Québec. Structure, évolution, réception, Montréal, Fides, 1988, 209 p.

3 Gérard Genette, Figures III, Paris, Seuil, 1972, p. 187, cité par Pierre Hébert, p. 25.

4 Le Journal de Saint-Denys Garneau commence en janvier 1935 et s'interrompt en janvier 1939; il a été publié en 1954. Pierre Hébert fait cependant courir la période du moi-recouvré de 1930 à 1950.

5 Yvan Lamonde, Je me souviens. La littérature personnelle au Québec, 18601980, Québec, IQRC, 1983, p. 15, cité par Pierre Hébert, p. 79.

6 Pour Didier, dans le joumal intime, il n'y a pas de récit. Le Journal intime, Paris, PUF, 1976, p. 140, cité par Pierre Hébert, p. 87-88.

7 Henriette Dessaulles, Fadette. Journal d'Henriette Dessaulles 1874-1880, Montréal, Hurtubise HMH, 1981.

8 Jean Rousset, «Le lecteur intime, texte sans destinataire?», le Lecteur intime, de Balzac au Journal, Paris, José Corti, 1986, p. 141-153.

9 Lionel Groulx, Journal 1895-1911, édition critique préparée par Gisèle Huot et Réjean Bergeron, Montréal, PUM, 1984, p. 784, cité par Pierre Hébert, p. 129.

10 Nicole Brossard, Jounal intime ou Voilà donc un manuscrit, Montréal, les Herbes rouges, 1984, p. 31, cité par Pierre Hébert, p. 23.

11 Robert Fothergill, Private Chronicles. A Study of English Diaries, London, Oxford University Press, 1974, p. 48, cité par Pierre Hébert, p. 84. 